\title{
Lack of Correlation between Dynamic Balance and Hamstring-to-Quadriceps Ratio in Patients with Chronic Anterior Cruciate Ligament Tears
}

\author{
Dae-Hee Lee, $\mathrm{MD}^{1,2}$, Jin-Hyuck Lee, $\mathrm{PT}^{2}$, Hye-Jin Jeong, $\mathrm{PT}^{2}$, and Seok-Joo Lee, $\mathrm{PT}^{2}$ \\ ${ }^{1}$ Department of Orthopaedic Surgery, Samsung Medical Center, Sungkyunkwan University School of Medicine, Seoul; ${ }^{2}$ Department of Sports Medical Center, Korea \\ University College of Medicine, Seoul, Korea
}

\begin{abstract}
Purpose: The purpose of this study was to evaluate the quadriceps and hamstring muscle strength and hamstring-to-quadriceps (HQ) ratio, as well as the relationships of these parameters with dynamic balance, in patients with anterior cruciate ligament (ACL) rupture.

Materials and Methods: We compared 25 patients diagnosed with chronic unilateral ACL tears and 25 age-matched healthy volunteers. The maximal torque of the quadriceps and hamstring and dynamic balance were measured.

Results: Although the isokinetic maximal peak torques were about $50 \%$ lower in the quadriceps $(57 \%, \mathrm{p}<0.001)$ and hamstring $(56 \%$, $\mathrm{p}=0.001)$ muscles in the chronic ACL tear group than in the control group, their HQ ratios were similar ( $56 \% \pm 17 \%$ vs. $58 \% \pm 6 \%, \mathrm{p}=0.591)$. HQ ratio was significantly correlated with anterior-posterior stability index $(r=-0.511, p=0.021)$ and overall stability index $(r=-0.476, p=0.034)$ in control group, but these correlations were not observed in chronic ACL tear group.

Conclusions: Thigh muscle strength was about 50\% lower in the chronic ACL tear group than in the control group, but the HQ ratio was similar. The dynamic balance of the knee was not influenced by thigh muscle strength but was influenced by HQ ratio in healthy young individuals. However, HQ ratio was not correlated with dynamic knee balance in chronic ACL tear patients.
\end{abstract}

Keywords: Anterior cruciate ligament, Chronic tear, Muscle strength, Postural balance

\section{Introduction}

Thigh muscles, including the quadriceps and hamstring muscles, adapt to injury to the anterior cruciate ligament (ACL) by losing strength ${ }^{1,2)}$. Decreased quadriceps muscle strength after ACL tear is regarded as universal ${ }^{3,4)}$ and is thought as a result of a compensating mechanism to prevent anterior subluxation ${ }^{5}$. Hamstring strength is also reduced following an ACL tear but

Received June 15, 2014; Revised (1st) November 26, 2014;

(2nd) January 19, 2015; Accepted February 9, 2015

Correspondence to: Dae-Hee Lee, MD

Department of Orthopaedic Surgery, Samsung Medical Center, 81 Irwonro, Gangnam-gu, Seoul 135-710, Korea

Tel: +82-2-3410-3507, Fax: +82-2-3410-0061

E-mail: eoak22@empal.com

This is an Open Access article distributed under the terms of the Creative Commons Attribution Non-Commercial License (http://creativecommons.org/licenses/by-nc/4.0/) which permits unrestricted non-commercial use, distribution, and reproduction in any medium, provided the original work is properly cited. the magnitude of the decrement is expected to be smaller than that of the quadriceps because ACL tears are often followed by physiological modifications that activate the hamstring to counteract anterior shear forces during knee loading ${ }^{6}$. Therefore, the balance between quadriceps and hamstring muscle strength, usually assessed as hamstring-to-quadriceps (HQ) ratio, may be different after ACL tears compared to that in normal individu$\mathrm{als}^{1,7,8)}$. Single-limb stance dynamic balance has been reported to be impaired in individuals with chronic ACL tears ${ }^{9,10)}$. Knee joint dynamic balance depends not only on the thigh muscle strength but also on the strength balance among thigh muscles groups ${ }^{11,12)}$. Therefore, weakening of the quadriceps and/or hamstring muscle strength and the resultant change in HQ ratio following ACL tears are expected to correlate with dynamic balance. However, few studies have investigated whether a thigh muscle deficit or HQ ratio is associated with dynamic balance in patients with ACL tears. We, therefore, compared the quadriceps and hamstring muscle strength and $\mathrm{HQ}$ ratio between patients with ACL tears and normal healthy individuals, as well as the relationships 
of these parameters with dynamic balance.

We hypothesized that the HQ ratio may differ between patients with chronic ACL tears and normal individuals, and, since muscle strength was reported not to correlate with postural stability ${ }^{13)}$, the HQ ratio may have a more significant effect on dynamic balance than the strength of the quadriceps or hamstring muscles.

\section{Materials and Methods}

\section{Study Design and Subjects}

This prospective longitudinal trial included male patients who were candidates for ACL reconstruction for chronic ACL tear on the dominant side between April 2009 and August 2010. Chronic ACL tear was defined as an ACL tear with an interval of

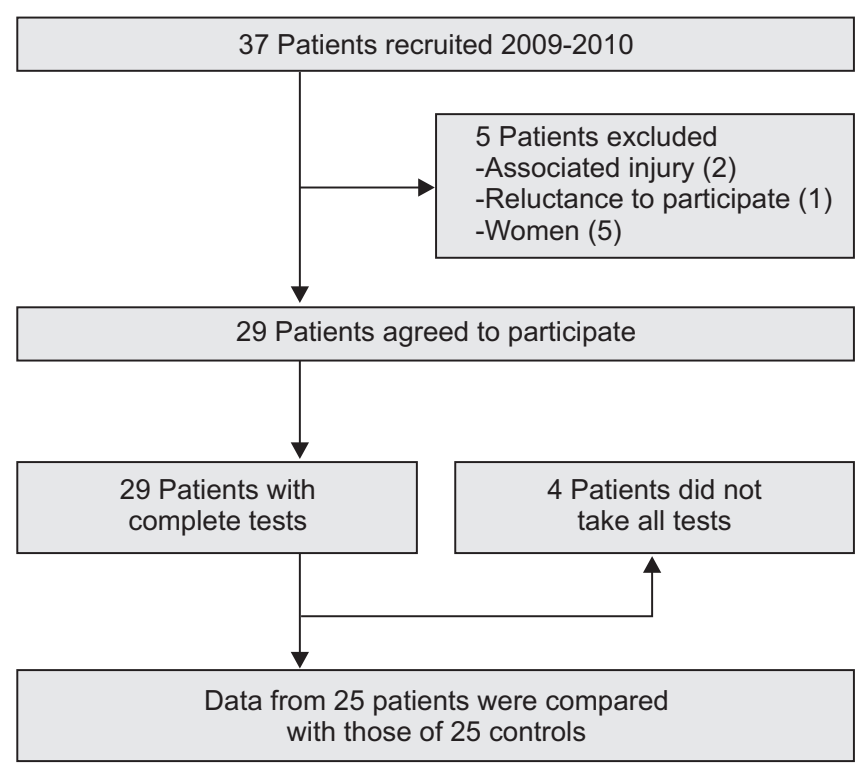

Fig. 1. Flowchart illustrating the study design.
$>3$ months from injury to testing (mean, 11.5 months; range, 4 months to 6 years) and confirmed by magnetic resonance imaging and physical examinations such as positive anterior drawer test, Lachmann test, and/or pivot shift test (more than grade I). Women were excluded to eliminate gender bias. We also excluded patients with bilateral injuries or other concomitant intra-articular injuries (i.e., meniscus or ligament injuries) or associated extra-articular lesions, those who were undergoing revisional ACL reconstruction, those who had a history of vestibular or neurologic dysfunction, and those with prior experience of dynamic balance testing or training using the Biodex Stability System (BSS; Biodex Medical Systems, Shirley, NY, USA) or force platform to exclude a learning curve effect.

Of the 37 patients ( 37 knees) initially enrolled for the study, 29 patients were included after eligibility assessment. The final analysis was based on data obtained from 25 patients, excluding 4 patients in whom pain or refusal to test resulted in incomplete data acquisition (Fig. 1). The 25 healthy male volunteers in the control group were selected from healthy control data collected by our institution. A 1:1 matched set design was employed, in which each ACL tear patient was matched to one control. The matching variables included age and level of sports activity. The physical activity level of the control group was similar to the preinjury level of the patients in the chronic ACL tear group. All 25 patients in the chronic ACL tear group had excellent (range, 95 to 100 points) Lysholm scores before the ACL tear, as did all 25 healthy male volunteers in the control group. Individuals in the control group had no history of vestibular or neurological problems, lower limb injuries or prior experience with balance testing or training. The baseline demographic characteristics of the two groups were similar (Table 1). The study protocol was approved by our Institutional Review Board. All subjects gave their written informed consent before participation.

Table 1. Demographic Characteristics of Subjects in the Chronic ACL tear Group and Control Group

\begin{tabular}{lccc}
\hline \multicolumn{1}{c}{ Parameter } & Chronic ACL tear group & Control group & p-value \\
\hline Sample size & 25 & 25 & 25 \\
Gender $(\mathrm{M})$ & 25 & $22.2 \pm 2.0(20-27)$ & 0.524 \\
Age $(\mathrm{yr})$ & $22.7 \pm 2.4(20-28)$ & $174.0 \pm 6.12(157-181)$ & 0.180 \\
Height $(\mathrm{cm})$ & $171.6 \pm 4.9(160-183)$ & $69.0 \pm 8.3(50-83)$ & 0.409 \\
Weight $(\mathrm{kg})$ & $71.2 \pm 8.4(61-92)$ & $22.7 \pm 2.1(18.3-25.9)$ & 0.093 \\
Body mass index $\left(\mathrm{kg} / \mathrm{m}^{2}\right)$ & $24.2 \pm 3.2(20.4-33.6)$ & 25 & 0.578 \\
Dominant \& injured side (right) & 25 & $5.4 \pm 1.1(3-9)$ & \\
Tegner activity scale & $5.3 \pm 1.2(3-8)$ & & \\
\hline
\end{tabular}

Values are presented as mean \pm standard deviation or number (range).

ACL: anterior cruciate ligament. 


\section{Assessment of Isokinetic Strength}

Isokinetic knee extension/flexion (concentric/concentric muscle action) strength was measured with each subject seated on a dynamometer with his trunk perpendicular to the floor, the hip flexed to 90 degrees, and the knee flexed to 90 degrees. Before each test session, each individual performed a warm-up set of 5 submaximal knee flexion/extensions for each leg at 60 degrees/ sec. Each test session consisted of 5 knee isokinetic extension/ flexion repetitions (range, 100 to 0 degrees) for each leg at 60 degrees/sec, with a rest time of 30 seconds between tests. Although both knees were tested in the two groups, the peak flexion and extension torques $(\mathrm{Nm} / \mathrm{kg})$ were recorded only on the dominant injured side in the ACL tear group and on the dominant side in the control group. Extensor strength was regarded as quadriceps strength, and flexor strength was regarded as hamstring muscle strength. Isokinetic concentric measurements of hamstrings and quadriceps strength demonstrate excellent reliability ${ }^{14)}$.

\section{Assessment of Dynamic Balance}

Dynamic balance tests were performed using the BSS, with a movable balance platform that provided up to $20^{\circ}$ of surface tilt in a $360^{\circ}$ range of motion. This platform, which interfaced with computer software (Biodex ver. 1.32, Biodex Medical Systems), enabled the device to objectively assess balance. Participants were instructed to stand with a bare foot on the BSS locked platform, to keep the other foot off the ground in a comfortable position, to keep arms at their sides and to look straight ahead at a point on the wall approximately $1 \mathrm{~m}$ away at eye level (Fig. 2). After completion of the centering process, which locked the platform and recorded the subject's foot position as a reference, the stability level automatically declined at every $1.66 \mathrm{sec}$ from level 12 (most stable) gradually to level 1 (least stable). The test consisted of two trials, each taking 20 seconds, with a 10 second rest between trials. The mean and standard deviation of the two trials was calculated by the stability system. The measures of balance and postural stability included anterior-posterior stability index (APSI), medial-lateral stability index (MLSI), and overall stability index (OSI) scores. These indices are standard deviations that assess the path of sway around the zero point from the center of the platform. APSI and MLSI represent foot displacement in the sagittal and coronal planes, respectively ${ }^{15,16)}$. The OSI score was established by combining the degree of the APSI and MLSI. All three indices were calibrated automatically by computer software. The lower the stability index, the more stable the platform, representing greater dynamic balance or postural stability of the subject. A high score in the indexes indicates poor balance.

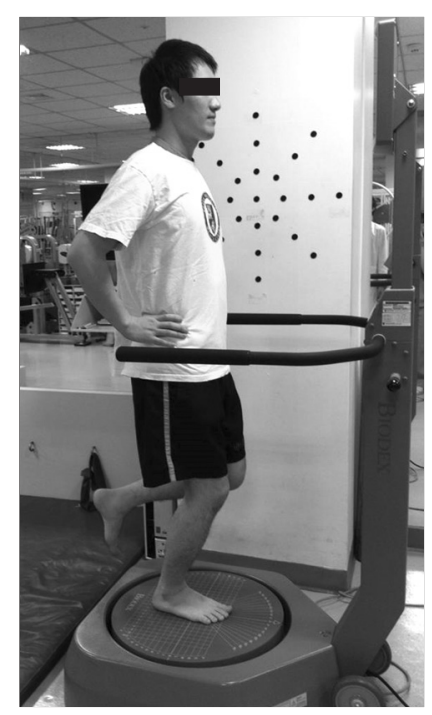

Fig. 2. Photograph of a subject demonstrating the measurement of dynamic balance using the Biodex Stability System.

\section{Statistical Analysis}

For comparison of the ACL tear and control group, we could estimate that the enrollment of 21 patients per group would give a statistical power of $80 \%$ using the Student's t-test with a significance level of 0.05 to detect a mean difference of $10 \%$ in the HQ ratio, assuming a pooled standard deviation of $11.2 \%$. Sample size estimation was based on our pilot study of 5 knees in each group. To achieve a greater power for the primary endpoint, we enrolled 25 patients in each group, resulting in an adequate power (0.845) to detect a significant difference between the two HQ ratios.

Statistical analysis was performed using SPSS ver. 12.00 (SPSS Inc., Chicago, IL, USA). Parametric variables were compared using Student's t-test and non-parametric variables were compared using Mann-Whitney U test, with a $\mathrm{p}<0.05$ considered statistically significant. Correlations between stability indices (OSI, APSI, and MLSI) and the strength of the hamstring or quadriceps muscle were assessed using Pearson's correlation analysis because all variables were continuous and parametric. Multiple linear regression analysis was performed to identify independent predictors of APSI, MLSI, and OSI in each group. Since postural stability has been associated with body mass index $(\mathrm{BMI})^{17)}$, and obesity and low muscle strength with aging have been associated with functional decline ${ }^{18)}$, age, height, weight, BMI, quadriceps and hamstring strength at $60 \% \mathrm{sec}$, and HQ ratio were included as potential independent variables. Candidate variables were selected for entry into the multiple linear regression model using a stepwise selection method, based on a significance level of $\mathrm{p}<0.05$. 
A

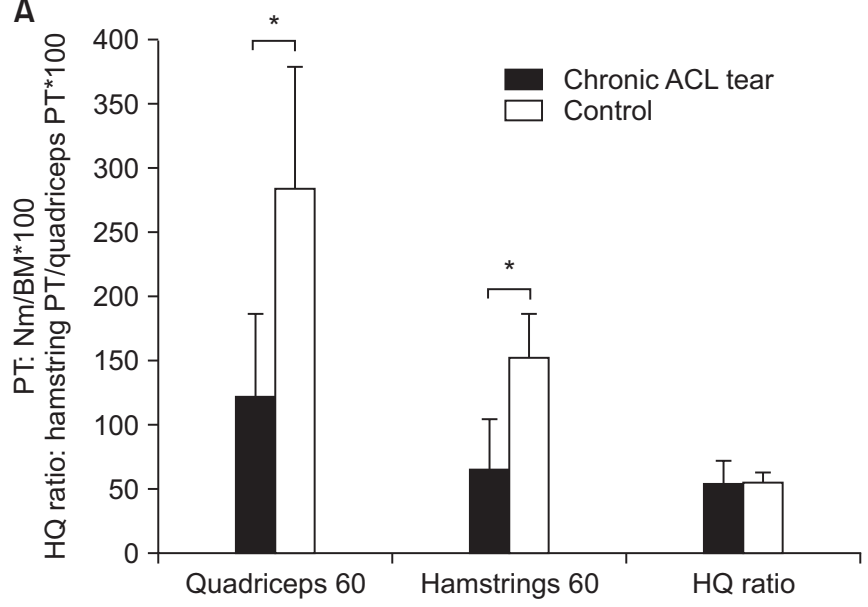

B

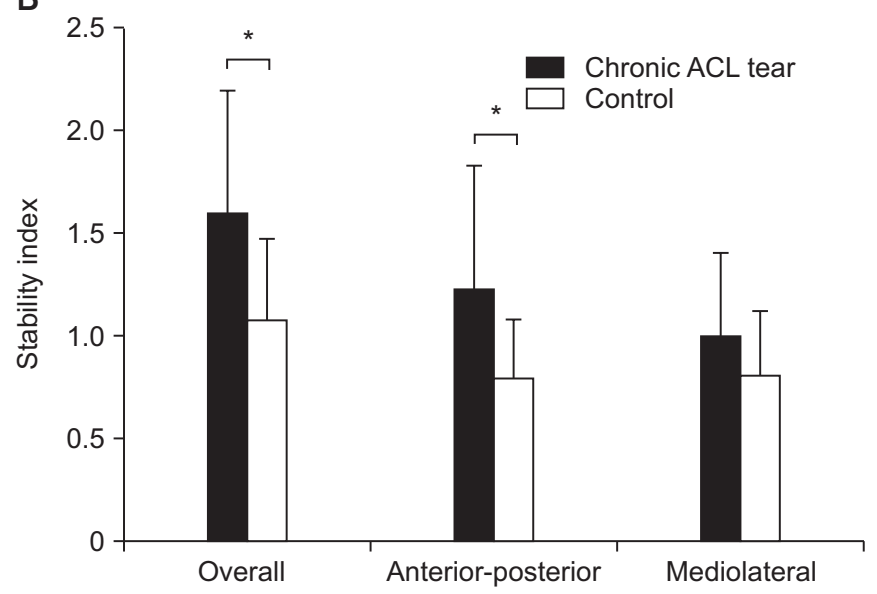

Fig. 3. (A) The mean isokinetic peak torque of both quadriceps and hamstrings, and the hamstring-to-quadriceps (HQ) peak torque ratio in the chronic ACL tear and control groups. (B) Stability indices for anterior-posterior, medial-lateral, and overall survival in the chronic ACL tear and control groups. All vertical error bars represent standard deviation. ACL: anterior cruciate ligament.

*Statistically significant.

\section{Results}

\section{Muscle Strength, HQ Ratio, and Dynamic Balance}

Although the isokinetic maximal peak torques were about $50 \%$ lower for the quadriceps $(57 \%, 122 \pm 65 \mathrm{Nm}$ vs. $284 \pm 95$ $\mathrm{Nm} ; \mathrm{p}<0.001)$ and hamstring $(56 \%, 68 \pm 38 \mathrm{Nm}$ vs. $152 \pm 34 \mathrm{Nm}$; $\mathrm{p}=0.001$ ) muscles in the chronic ACL tear group than those in the control group, their HQ ratios were not significantly different $(56 \% \pm 17 \%$ vs. $58 \% \pm 6 \%, p=0.591)$ (Fig. $3 \mathrm{~A})$. The mean OSI and APSI scores were $44 \%(1.60 \pm 0.64$ vs. $1.08 \pm 0.40, \mathrm{p}=0.004)$ and $46 \%$ ( $1.23 \pm 0.56$ vs. $0.79 \pm 0.29, \mathrm{p}=0.003)$ higher, respectively, in the chronic ACL tear group than in the control group. Although the mean MLSI was 28\% higher in the chronic ACL tear group than in the control group, this difference was not statistically significant $(1.00 \pm 0.43$ vs. $0.82 \pm 0.33, \mathrm{p}=0.157)$ (Fig. $3 \mathrm{~B}$ ).

\section{Correlations and Predictor of Dynamic Balance}

The HQ ratio showed significantly negative correlations with OSI $(\mathrm{r}=-0.476, \mathrm{p}=0.034)$ and APSI $(\mathrm{r}=-0.511, \mathrm{p}=0.021)$ in the control group, but not in the chronic ACL tear group. The HQ ratio, however, was not correlated with MLSI in either group. We also found that neither quadriceps nor hamstring peak torque at $60^{\circ} / \mathrm{sec}$ was correlated with OSI, APSI, and MLSI in either group (Table 2). In the chronic ACL tear group, there was no correlation between the duration of ACL injury and any measure of muscle strength or stability $(\mathrm{p}>0.05)$. The multiple regression analysis showed that the HQ ratio was the only significant predictor of APSI and OSI in the control group, but we could not identify factors significantly predicting MLSI in this group (Table 3). None
Table 2. Correlations between Muscle Strength, HQ Ratio, and Dynamic Balance in the Chronic ACL Tear Group and Control Group

\begin{tabular}{lcccccc}
\hline \multirow{2}{*}{\multicolumn{1}{c}{ Parameter }} & \multicolumn{2}{c}{$\begin{array}{c}\text { Chronic ACL } \\
\text { tear group }\end{array}$} & & \multicolumn{2}{c}{$\begin{array}{c}\text { Control } \\
\text { group }\end{array}$} \\
\cline { 2 - 3 } \cline { 6 - 7 } & PCC $(\mathrm{r})$ & $\mathrm{p}$-value & & PCC (r) & p-value \\
\hline Quadriceps 60/OSI & -0.106 & 0.655 & & 0.382 & 0.097 \\
Quadriceps 60/APSI & -0.164 & 0.491 & & 0.330 & 0.156 \\
Quadriceps 60/MLSI & -0.193 & 0.416 & & 0.023 & 0.924 \\
Hamstrings 60/OSI & -0.181 & 0.445 & & 0.108 & 0.649 \\
Hamstrings 60/APSI & -0.265 & 0.259 & & 0.085 & 0.722 \\
Hamstrings 60/MLSI & -0.212 & 0.370 & & -0.140 & 0.557 \\
HQ ratio/OSI & -0.384 & 0.133 & & -0.476 & $0.034^{\mathrm{a})}$ \\
HQ ratio/APSI & -0.347 & 0.134 & & -0.511 & $0.021^{\mathrm{a})}$ \\
HQ ratio/MLSI & -0.197 & 0.404 & & -0.291 & 0.213 \\
\hline
\end{tabular}

HQ: hamstring-to-quadriceps, ACL: anterior cruciate ligament, PCC: Pearson correlation coefficient, OSI: overall stability index, APSI: anterior-posterior stability index, MLSI: medial-lateral stability index.

${ }^{\text {a) }}$ Statistically significant.

of the analyzed factors was a significant predictor of APSI, MLSI, or OSI in the chronic ACL tear group.

\section{Discussion}

We analyzed the relationship between HQ ratio and dynamic balance in patients with chronic ACL tears and control healthy individuals using quantitative methods such as isokinetic muscle strength tests and the stability index of BSS. The primary finding 
Table 3. Multiple Linear Regression Analysis to Identify Factors Affecting Stability Indices of the Control Group

\begin{tabular}{|c|c|c|c|c|c|c|}
\hline \multirow{2}{*}{ Dependent variable } & \multirow{2}{*}{$\begin{array}{l}\text { Explicative } \\
\text { variable }\end{array}$} & \multicolumn{2}{|c|}{ Unstandardized coefficients } & \multicolumn{3}{|c|}{ Standardized coefficients } \\
\hline & & $\mathrm{B}$ & SE (B) & $\beta$ & p-value & $\mathrm{R}^{2}$ \\
\hline OSI & HQ ratio (\%) & -0.030 & 0.014 & -0.458 & 0.042 & 0.210 \\
\hline APSI & HQ ratio (\%) & -0.023 & 0.010 & -0.479 & 0.033 & 0.229 \\
\hline
\end{tabular}

SE: standard error, OSI: overall stability index, HQ: hamstring-to-quadriceps, APSI: anterior-posterior stability index.

of this study was that the HQ ratio was significantly associated with dynamic balance (OSI and APSI) in the normal control group but not in the ACL tear group.

The isokinetic quadriceps muscle strength was shown to be significantly weaker in knees with ACL tears than in the contralateral uninjured knees ${ }^{19)}$ or in those of normal healthy controls ${ }^{20)}$. However, conflicting findings have been reported regarding hamstring muscle strength deficit in knees with ACL tears ${ }^{20}$. The quadriceps muscle may be affected by post-injury neural inhibition due to the loss of afferent feedback from the ACL to gamma motoneurons, resulting in a quadriceps avoidance gait pattern to prevent anterior subluxation of the tibia ${ }^{6,21)}$. Following ACL tears, however, the deficits in strength are not as large in hamstrings as in quadriceps muscles ${ }^{22)}$ and even 6 months after injury in a subconscious attempt to counteract anterior shear forces ${ }^{3)}$.

Although HQ ratio was expected to increase slightly in ACL tear patients due to greater deficits in quadriceps than hamstring strength, HQ ratios have been reported to be similar in these patients and in healthy controls. For example, a comparison of isokinetic muscle strength in the injured and uninjured knees of 18 subjects with unilateral chronic ACL deficient found that the HQ ratio was slightly higher in the injured than in the uninjured limbs, but the difference was not statistically significant $(71 \% \pm 13 \% \text { vs. } 65 \% \pm 13 \%, \mathrm{p}=0.205)^{222}$. Similarly, a comparison of 10 knees with complete ACL ruptures awaiting reconstructive surgery and uninjured knees found no difference in $\mathrm{HQ}$ ratio $(47 \% \pm 11 \% \text { vs. } 50 \% \pm 5 \%, p>0.05)^{23)}$. In agreement with these findings, we also observed no significant difference in HQ ratio between the chronic ACL tear group and normal control group $(56 \% \pm 17 \%$ vs. $58 \% \pm 6 \%, \mathrm{p}=0.591)$. This similar HQ ratio of the chronic ACL tear group indicates that the hamstrings in the ACL tear patients lack sufficient strength to serve as a dynamic agonist of the $\mathrm{ACL}^{8)}$ and compensate for functional deficits in the ACL to prevent anterior tibial translation ${ }^{22)}$

We also found that dynamic balance (OSI and APSI) was worse in ACL tear patients than in normal controls. Similarly, a comparison of $32 \mathrm{ACL}$-deficient knees and 57 healthy young volunteers found that dynamic stability was better in the control than in the ACL tear group during one-leg standing ${ }^{24)}$. The poorer dynamic stability in knees with ACL tears may be explained partly by the inability of the primary reflex arc from the ACL to the hamstring muscle (ACL-hamstring reflex arc) to play a role in joint stability. Rather, only the secondary reflex arc (joint capsule-hamstring reflex arc), mediated by stretch receptors in the hamstring muscle spindle and joint capsule, can regulate knee joint stability following damage to the ACL. This secondary reflex arc is unable to provide sufficient joint stability, especially when poorly exercised muscles surround the knee joints ${ }^{25)}$.

We also found that the correlation between HQ ratio and dynamic balance observed in the normal control group was not observed in the chronic ACL tear group; that HQ ratio was the only predictor of dynamic balance; and that muscle strength was not associated with dynamic balance in both groups. Despite the abundant isokinetic data on ACL deficient knees, only two previous studies have attempted to correlate isokinetic data with dynamic balance. In 40 male patients with ACL-deficient knees prior to reconstruction, dynamic balance using the BSS was associated with peak torques of quadriceps and hamstrings ${ }^{26)}$ contrary to our findings. Another study found that the strength of the quadriceps and hamstring muscles, as well as HQ ratio, were not associated with dynamic balance in ACL-deficient knees, although a significant correlation was observed between HQ ratio and dynamic balance in uninjured limbs ${ }^{27}$. These findings were similar to the results of our study, except that we used healthy young volunteers instead of the uninjured limb as controls. However, direct between-study comparisons are difficult due to disparities in study design, and methods for measuring dynamic balance including knee position ${ }^{28}$, and inter-subject variation especially regarding the chronicity of ACL tear or pre-injured sports activity level. In regard to the chronicity of ACL tear, we observed no correlation between the duration of ACL injury and any measure of muscle strength or stability.

Interestingly, we found that mean OSI and APSI were greater in the chronic ACL tear group than in the control group, although there was no intergroup difference in MLSI. We also found that the HQ ratio was significantly associated with OSI and APSI, but not with MLSI, in the control group. These findings may be due, at least in part, to the greater magnitude of motion in the 
anteroposterior than in the mediolateral direction, which may be caused by the greater gravitational torque around the AP than around the mediolateral motion axis ${ }^{29)}$. These findings indicate that separate determinations of APSI and MLSI would be better than the combined form of OSI ${ }^{30)}$. Because MLSI contributes a relatively small portion to the OSI, clinically important mediolateral instabilities might be overlooked if only the OSI were used.

This study had several limitations. The imbalance between knee flexors and extensors was assessed as the conventional concentric HQ ratio. However, because the opposing muscles cannot simultaneously contract concentrically, the "functional HQ ratio" between maximal eccentric knee flexion strength and maximal concentric knee extension strength may better reflect functional differences between hamstring and quadriceps strengths than does the concentric HQ ratio. In addition, measurements of muscle strength, such as muscle onset time, muscle contraction time, and muscle endurance, may be more important in maintaining dynamic balance than is muscle strength. There is also a limit to the generalizability of our results because we focused on young males. We assessed only male subjects in this study to avoid any gender difference in maximum performance caused by difference in sensitivity to pain. Another limitation is the discrepancy between the mechanisms underlying tests of muscle strength and dynamic balance. As one-leg standing is a closed-chain movement and the isokinetic knee muscle strength test involves an open-chain movement, the mechanism of muscle performance may differ between subjects with chronic ACL injury and healthy controls. This discrepancy may explain, at least in part, the lack of correlation between HQ ratio and stability index in our chronic ACL tear group. We also could not exclude the possibility of potential visual compensation for decreased contribution of the injured ACL to dynamic balance ${ }^{24)}$. Although the subjects kept their eyes open, the control screen was covered, thus preventing visual feedback regarding their performance during the dynamic balance test. These effects may decrease the bias caused by visual compensation.

Finally, we used post-trauma 3 months as the time point to distinguish between acute and chronic ACL tears. However, previous studies used different differentiating time points, ranging from 6 weeks to 6 months after trauma.

\section{Conclusions}

In conclusion, thigh muscle strength was about 50\% lower in the chronic ACL group than in the control group, but the HQ ratio was similar. We also found that the dynamic balance of the knee was not influenced by thigh muscle strength but was influenced by HQ ratio in healthy young males. However, the correlation between the HQ ratio and dynamic knee balance was not observed in patients with chronic ACL tears.

\section{Conflict of Interest}

No potential conflict of interest relevant to this article was reported.

\section{References}

1. Hiemstra LA, Webber S, MacDonald PB, Kriellaars DJ. Hamstring and quadriceps strength balance in normal and hamstring anterior cruciate ligament-reconstructed subjects. Clin J Sport Med. 2004;14:274-80.

2. Seo SS, Kim CW, Kim JG, Jin SY. Clinical results comparing transtibial technique and outside in technique in single bundle anterior cruciate ligament reconstruction. Knee Surg Relat Res. 2013;25:133-40.

3. de Jong SN, van Caspel DR, van Haeff MJ, Saris DB. Functional assessment and muscle strength before and after reconstruction of chronic anterior cruciate ligament lesions. Arthroscopy. 2007;23:21-8,

4. Konishi Y, Fukubayashi T, Takeshita D. Mechanism of quadriceps femoris muscle weakness in patients with anterior cruciate ligament reconstruction. Scand J Med Sci Sports. 2002;12:371-5.

5. Papadonikolakis A, Cooper L, Stergiou N, Georgoulis AD, Soucacos PN. Compensatory mechanisms in anterior cruciate ligament deficiency. Knee Surg Sports Traumatol Arthrosc. 2003;11:235-43.

6. Georgoulis AD, Ristanis S, Moraiti CO, Paschos N, Zampeli F, Xergia S, Georgiou S, Patras K, Vasiliadis HS, Mitsionis G. ACL injury and reconstruction: clinical related in vivo biomechanics. Orthop Traumatol Surg Res. 2010;96(8 Suppl): S119-28.

7. Costa PB, Ryan ED, Herda TJ, DeFreitas JM, Beck TW, Cramer JT. Effects of stretching on peak torque and the H:Q ratio. Int J Sports Med. 2009;30:60-5.

8. Hewett TE, Myer GD, Zazulak BT. Hamstrings to quadriceps peak torque ratios diverge between sexes with increasing isokinetic angular velocity. J Sci Med Sport. 2008;11:452-9.

9. Gauffin H, Pettersson G, Tegner Y, Tropp H. Function testing in patients with old rupture of the anterior cruciate ligament. Int J Sports Med. 1990;11:73-7. 
10. Lysholm M, Ledin T, Odkvist LM, Good L. Postural control: a comparison between patients with chronic anterior cruciate ligament insufficiency and healthy individuals. Scand J Med Sci Sports. 1998;8:432-8.

11. White KK, Lee SS, Cutuk A, Hargens AR, Pedowitz RA. EMG power spectra of intercollegiate athletes and anterior cruciate ligament injury risk in females. Med Sci Sports Exerc. 2003;35:371-6.

12. Park JS, Nam DC, Kim DH, Kim HK, Hwang SC. Measurement of knee morphometrics using MRI: a comparative study between ACL-injured and non-injured knees. Knee Surg Relat Res. 2012;24:180-5.

13. Ageberg E, Roberts D, Holmstrom E, Friden T. Balance in single-limb stance in patients with anterior cruciate ligament injury: relation to knee laxity, proprioception, muscle strength, and subjective function. Am J Sports Med. 2005;33: 1527-35.

14. Myer GD, Ford KR, Barber Foss KD, Liu C, Nick TG, Hewett TE. The relationship of hamstrings and quadriceps strength to anterior cruciate ligament injury in female athletes. Clin J Sport Med. 2009;19:3-8.

15. Arifin N, Abu Osman NA, Wan Abas WA. Intrarater testretest reliability of static and dynamic stability indexes measurement using the biodex stability system during unilateral stance. J Appl Biomech. 2014;30:300-4.

16. Mattacola CG, Perrin DH, Gansneder BM, Gieck JH, Saliba EN, McCue FC 3rd. Strength, functional outcome, and postural stability after anterior cruciate ligament reconstruction. J Athl Train. 2002;37:262-268.

17. Greve J, Alonso A, Bordini AC, Camanho GL. Correlation between body mass index and postural balance. Clinics (Sao Paulo). 2007;62:717-20.

18. Hegeman J, Shapkova EY, Honegger F, Allum JH. Effect of age and height on trunk sway during stance and gait. J Vestib Res. 2007;17:75-87.

19. Keays SL, Bullock-Saxton J, Keays AC, Newcombe P. Muscle strength and function before and after anterior cruciate ligament reconstruction using semitendonosus and gracilis. Knee. 2001;8:229-34.

20. Patel RR, Hurwitz DE, Bush-Joseph CA, Bach BR Jr, Andri- acchi TP. Comparison of clinical and dynamic knee function in patients with anterior cruciate ligament deficiency. Am J Sports Med. 2003;31:68-74.

21. Tsepis E, Vagenas G, Ristanis S, Georgoulis AD. Thigh muscle weakness in ACL-deficient knees persists without structured rehabilitation. Clin Orthop Relat Res. 2006;450:211-8.

22. St Clair Gibson A, Lambert MI, Durandt JJ, Scales N, Noakes TD. Quadriceps and hamstrings peak torque ratio changes in persons with chronic anterior cruciate ligament deficiency. J Orthop Sports Phys Ther. 2000;30:418-27.

23. Hole CD, Smit GH, Hammond J, Kumar A, Saxton J, Cochrane T. Dynamic control and conventional strength ratios of the quadriceps and hamstrings in subjects with anterior cruciate ligament deficiency. Ergonomics. 2000;43:1603-9.

24. Okuda K, Abe N, Katayama Y, Senda M, Kuroda T, Inoue $\mathrm{H}$. Effect of vision on postural sway in anterior cruciate ligament injured knees. J Orthop Sci. 2005;10:277-83.

25. Solomonow M, Baratta R, Zhou BH, Shoji H, Bose W, Beck C, D'Ambrosia R. The synergistic action of the anterior cruciate ligament and thigh muscles in maintaining joint stability. Am J Sports Med. 1987;15:207-13.

26. Park WH, Kim DK, Yoo JC, Lee YS, Hwang JH, Chang MJ, Park YS. Correlation between dynamic postural stability and muscle strength, anterior instability, and knee scale in anterior cruciate ligament deficient knees. Arch Orthop Trauma Surg. 2010;130:1013-8.

27. Lee HM, Cheng CK, Liau JJ. Correlation between proprioception, muscle strength, knee laxity, and dynamic standing balance in patients with chronic anterior cruciate ligament deficiency. Knee. 2009;16:387-91.

28. Pereira HM, de Campos TF, Santos MB, Cardoso JR, Garcia Mde C, Cohen M. Influence of knee position on the postural stability index registered by the biodex stability system. Gait Posture. 2008;28:668-72.

29. Paterno MV, Myer GD, Ford KR, Hewett TE. Neuromuscular training improves single-limb stability in young female athletes. J Orthop Sports Phys Ther. 2004;34:305-16.

30. Arnold BL, Schmitz RJ. Examination of balance measures produced by the biodex stability system. J Athl Train. 1998;33:323-7. 